\title{
A Library of Quinoline-labeled Water-soluble Copolymers with pH-Tunable Fluorescence Response in the Acidic pH Region
}

Ioannis Thivaios $†$, Sofia Kakogianni $†$ and Georgios Bokias $†, \neq *$

${ }^{\dagger}$ Department of Chemistry, University of Patras, University Campus, Rio-Patras, GR26504, Greece

${ }^{\ddagger}$ Foundation for Research and Technology Hellas / Institute of Chemical Engineering Sciences (FORTH/ICE-HT), Platani Str., Patras, GR26504, Greece

Synthesis of bromobenzimidazole:In a round bottom flask of $1000 \mathrm{ml}$, where a magnet and an ice bath were placed, was added $150 \mathrm{~g} \mathrm{KOH}$ and $400 \mathrm{ml} \mathrm{MeOH}$. Alongside in a flask of $250 \mathrm{ml}$ with a magnet $25 \mathrm{~g}$ p-bromonitrobenzene and $100 \mathrm{ml}$ THF were added. After the solids were completely dissolved in both flasks, the $1000 \mathrm{ml}$ flask was placed in a renewed ice bath and the solution of the $250 \mathrm{ml}$ flask was added through filter paper to contain any impurities. A condenser was adapted and degassing cycles and Ar/vacuum took place. The system remained under $\mathrm{Ar}$ atmosphere for $\sim 15 \mathrm{~min}$. Then $17 \mathrm{ml}$ of benzylcyanide were slowly added and the reaction was left in an ice bath for $\sim 3$ hours with continuous renewal of ice. The content of the flask was 
precipitated in $\mathrm{H}_{2} \mathrm{O}$ and then filtered under vacuum. The precipitate was dried at $75^{\circ} \mathrm{C}$ for 2hours and then recrystallized in $\mathrm{MeOH}$. Filtration on porous filter without vacuum followed recrystallization and the pure material was dried at $50^{\circ} \mathrm{C}$ under vacuum for $\sim 12$ hours.

Synthesis of 2-amino-5-bromo-benzophenone: In a round bottom of 500ml flask where a condenser, magnetic stirring and oil bath were placed, was added $6 \mathrm{~g}$ bromobenzimidazole and $\sim 150 \mathrm{ml}$ of acetic acid and the mixture heated at $110^{\circ} \mathrm{C}$. Addition of $4.5 \mathrm{~g}$ Fe and $10 \mathrm{ml} \mathrm{H}_{2} \mathrm{O}$ every 10min was followed. The temperature was removed upon the third addition. After the solution was cooled to ambient temperature, $500 \mathrm{ml} \mathrm{H}_{2} \mathrm{O}$ was added followed by filtration over a fluted filter to contain iron and washed with ethyl acetate. The filtrate was extracted with EtOAc (3x $40 \mathrm{ml})$ and ultra-pure water $(3 \mathrm{x} 40 \mathrm{ml})$. The organic phase was stirred with magnesium sulfate $\left(\mathrm{MgSO}_{4}\right)$, then filtered and rotary concentrated in vacuum (rotary evaporation) at $55^{\circ} \mathrm{C}$. The purified solid was dried in vacuum for 12 hours.

Synthesis of 6-bromo-2-phenyl-4-phenylquinoline: In a round bottom flask of 100ml, where a condenser, magnetic stirring and oil bath were placed, degassing cycles and Ar/vacuum were performed. The flask was cooled under Ar and then $1.75 \mathrm{~g}(6.34 \mathrm{mmol})$ 2-amino- bromobenzophenone and $20 \mathrm{ml}$ of acetic acid $(\mathrm{AcOH})$ were added and the mixture was heated at $110^{\circ} \mathrm{C}$. When the 2-amino-5-bromobenzophenone dissolved $2 \mathrm{~g}(9.52 \mathrm{mmol})$ of acetophenone and $1 \mathrm{ml}$ of sulfuric acid $95 \%\left(\mathrm{H}_{2} \mathrm{SO}_{4}\right)$ was added to the reaction. The flask was degassed and filled with argon and heated to reflux for 5 days. Then the solution was cooled and precipitated into a mixture of ice/water/ammonia in the ratio of $1 / 1 / 0.2$. The mixture was filtered and dried under vacuum at $60^{\circ} \mathrm{C}$ for 12 hours. The obtained solid was dispersed in $25 \mathrm{ml}$ of absolute ethanol for further purification. The pure solid was filtered and dried under vacuum for 12 hours. 
The 6-bromo-2-cyanophenyl-4-phenylquinoline and 6-bromo-2-perfluorophenyl-4phenylquinoline were synthesized according to previous methodology.

Synthesis of the vinyl monomer 2,4-diphenyl-6-(4-vinylphenyl)quinoline (SDPQ): In a round bottom flask of $100 \mathrm{ml}$, where a condenser, magnetic stirring and oil bath were placed, degassing cycles and $\mathrm{Ar} / \mathrm{vacuum}$ were performed. The flask was cooled under Ar and then $2 \mathrm{~g}(4.44 \mathrm{mmol})$ $\mathrm{BrDPQ}$, 0.86g (5.78mmol) 4-vinylphenylboronic acid, $1.84 \mathrm{~g}(13.32 \mathrm{mmol})$ in $2 \mathrm{M}$ aqueous $\mathrm{K}_{2} \mathrm{CO}_{3}$ solution, $0.15 \mathrm{~g}(0.13 \mathrm{mmol}) \mathrm{Pd}\left(\mathrm{PPh}_{3}\right)_{4}$ and $50 \mathrm{~mL}$ of toluene were added and the flask was degassed and it was filled again with Ar. The flask was covered with aluminum foil and the reaction mixture was heated to reflux for 4days. The mixture was filtered through a fluted filter, followed by extraction of the organic layer with EtOAc $(3 \mathrm{x} 40 \mathrm{ml})$ and ultra-pure water $(3 \mathrm{x}$ $40 \mathrm{ml})$. The organic phase was stirred with magnesium sulfate $\left(\mathrm{MgSO}_{4}\right)$, then filtered and rotary concentrated in vacuum (rotary evaporation). The solid was dried under vacuum at $40^{\circ} \mathrm{C}$ for 12hours and then was dispersed in methanol for further purification. The purified yellow solid was filtered and dried under vacuum for 12 hours.

The 6-(4-vinylphenyl)-2-cyanophenyl-4-phenylquinoline (SDPQCN) and the 6-(4vinylphenyl)-2-pentafluorophenyl-quinoline (5FSPQ) were synthesized according to previous methodology.

Synthesis of the watersoluble P(AMPSA-SDPQ) copolymers: In a round bottom flask of $250 \mathrm{ml}$, where a condenser, magnetic stirring and oil bath were placed, degassing cycles and Ar/vacuum were performed. The flask was cooled under Ar and then $10 \mathrm{~g}(0.05 \mathrm{~mol})$ AMPSA, $0.040 \mathrm{~g}\left(1 * 10^{-4}\right.$ mol) SDPQ, $0.082 \mathrm{~g}\left(5^{*} 10^{-4} \mathrm{~mol}\right)$ AIBN and finally $100 \mathrm{ml}$ DMF were added and the flask was degassed and it was filled with Ar again. The reaction mixture was heated at $110^{\circ} \mathrm{C}$ for 24 hours. 
After cooling down to room temperature the resulting copolymers were precipitated in EtOAc and filtrated. Then, the crude copolymer was dissolved in ultra-pure water and there it was changed into the sodium salt form through neutralization with an excess of $\mathrm{NaOH}$. Then the copolymer was further purified through dialysis (membrane cut-off: 12kDa), which was immersed in a large excess of water at $22^{\circ} \mathrm{C}$ for several days by replacing the water twice a day, monitoring the $\mathrm{pH}$ and the conductivity until it was similar to the values of the deionized water. The membrane then was removed and the copolymer solution was freeze-dried, collected and stored in a desiccator.

The synthesis of the watersoluble P(AMPSA-SDPQCN) and P(AMPSA-5FSPQ) copolymers were synthesized according to previous methodology using the same reagents' ratios.

Synthesis of the watersoluble P(DMAM-SDPQ) copolymer: In a round bottom flask of $250 \mathrm{ml}$, where a condenser, magnetic stirring and oil bath were placed, degassing cycles and $\mathrm{Ar} / \mathrm{vacuum}$ were performed. The flask was cooled under Ar and then $10 \mathrm{~g}(0.10 \mathrm{~mol})$ DMAM, $0.080 \mathrm{~g}\left(2 * 10^{-4}\right.$ mol) SDPQ, $0.17 \mathrm{~g}\left(1 * 10^{-3} \mathrm{~mol}\right)$ AIBN and finally $100 \mathrm{~mL}$ THF were added and the flask was degassed and it was filled with Ar again. The reaction mixture was refluxed for 24hours. After cooling down to room temperature the resulting copolymers were precipitated in $\mathrm{Et}_{2} \mathrm{O}$ and filtrated. Then, the crude copolymer was dissolved in ultra-pure water and further purified through dialysis (membrane cut-off: $12 \mathrm{kDa}$ ), which was immersed in a large excess of water at $22^{\circ} \mathrm{C}$ for several days by replacing the water twice a day, monitoring the conductivity, until it was similar to the value of the deionized water. The membrane then was removed and the copolymer solution was freeze-dried, collected and stored in a desiccator. 
The synthesis of the watersoluble $\underline{\mathrm{P}(\mathrm{DMAM}-\mathrm{SDPQCN})}$ and $\underline{\mathrm{P}(\mathrm{DMAM}-5 \mathrm{FSPQ})}$ copolymers were synthesized according to previous methodology using the same reagents' ratios.

Synthesis of the watersoluble P(MAPTAC-SDPQ) copolymer: In a round bottom flask of 250ml, where a condenser, magnetic stirring and oil bath were placed, degassing cycles and Ar/vacuum were performed. The flask was cooled under Ar and then $20 \mathrm{ml}(0.045 \mathrm{~mol})$ of $50 \%$ wt MAPTAC in $\mathrm{H}_{2} \mathrm{O}, 0.036 \mathrm{~g}\left(9^{*} 10^{-5} \mathrm{~mol}\right) \mathrm{SDPQ}, 0.074 \mathrm{~g}\left(4.5^{*} 10^{-4} \mathrm{~mol}\right)$ AIBN and finally $100 \mathrm{ml}$ DMF were added and the flask was degassed and it was filled with Ar again. The reaction mixture was heated at $110^{\circ} \mathrm{C}$ for 24 hours. After cooling down to room temperature the resulting copolymers were precipitated in EtOAc and filtrated. Then, the crude copolymer was dissolved in ultra-pure water and further purified through dialysis (membrane cut-off: $12 \mathrm{kDa}$ ), which was immersed in a large excess of water at $22^{\circ} \mathrm{C}$ for several days by replacing the water twice a day, monitoring the $\mathrm{pH}$ and the conductivity, until it was similar to the values of the deionized water. The membrane then was removed and the copolymer solution was freeze-dried, collected and stored in a desiccator. 


\section{${ }^{1}$ H NMR characterization of monomers and copolymers}

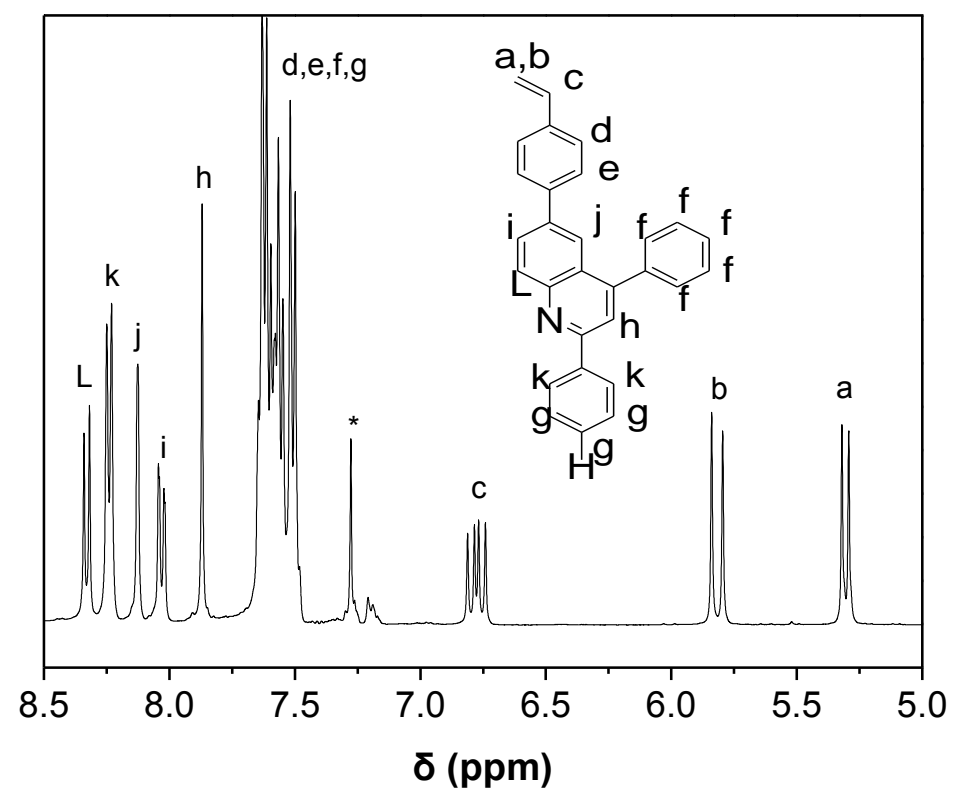

Figure S1. ${ }^{1} \mathrm{H}$ NMR spectrum of SDPQ in $\mathrm{CDCl}_{3}$.

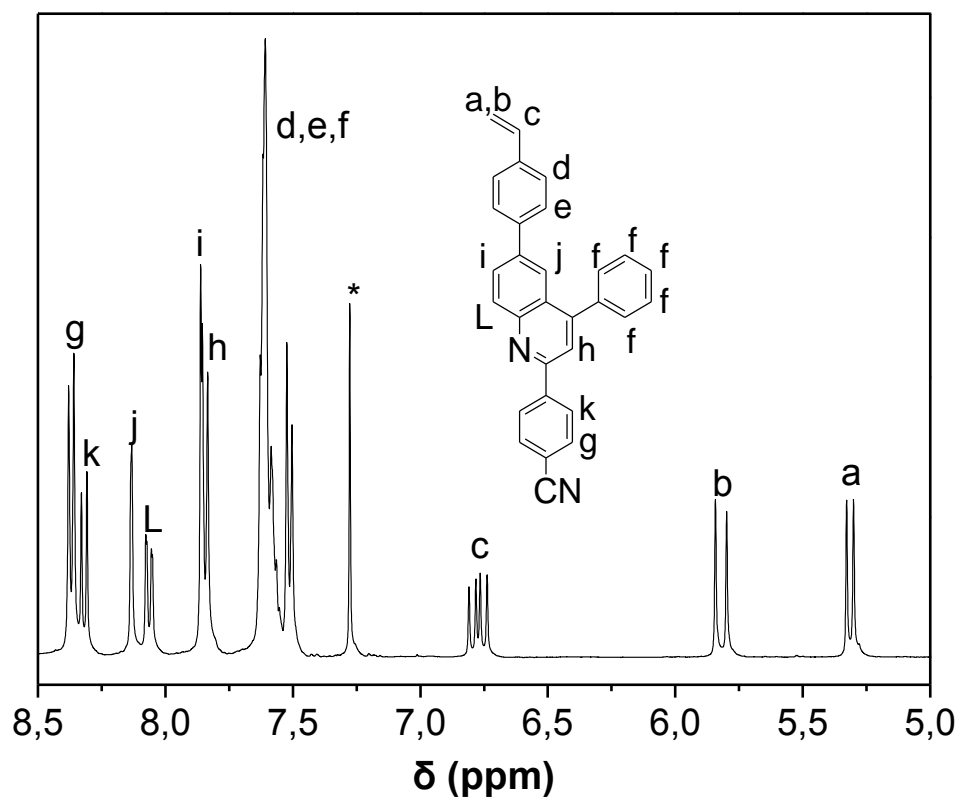

Figure S2. ${ }^{1} \mathrm{H} \mathrm{NMR}$ spectrum of SDPQCN in $\mathrm{CDCl}_{3}$. 


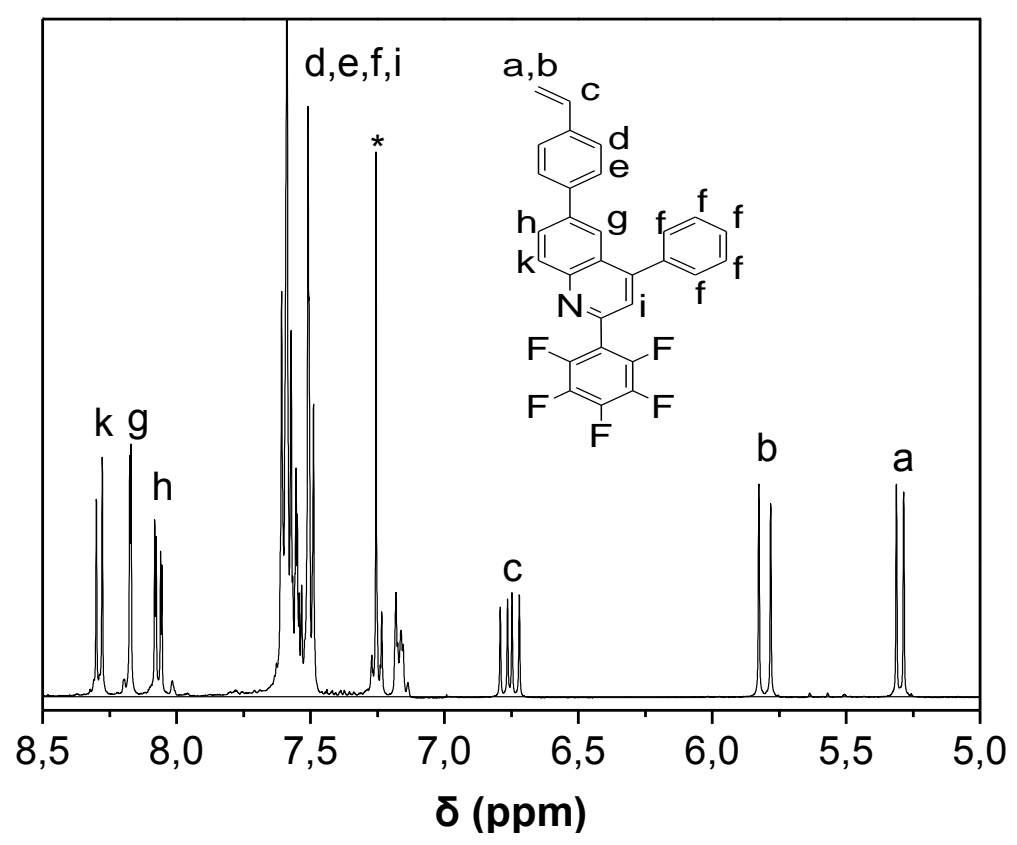

Figure S3. ${ }^{1} \mathrm{H}$ NMR spectrum of $5 \mathrm{FSPQ}$ in $\mathrm{CDCl}_{3}$.

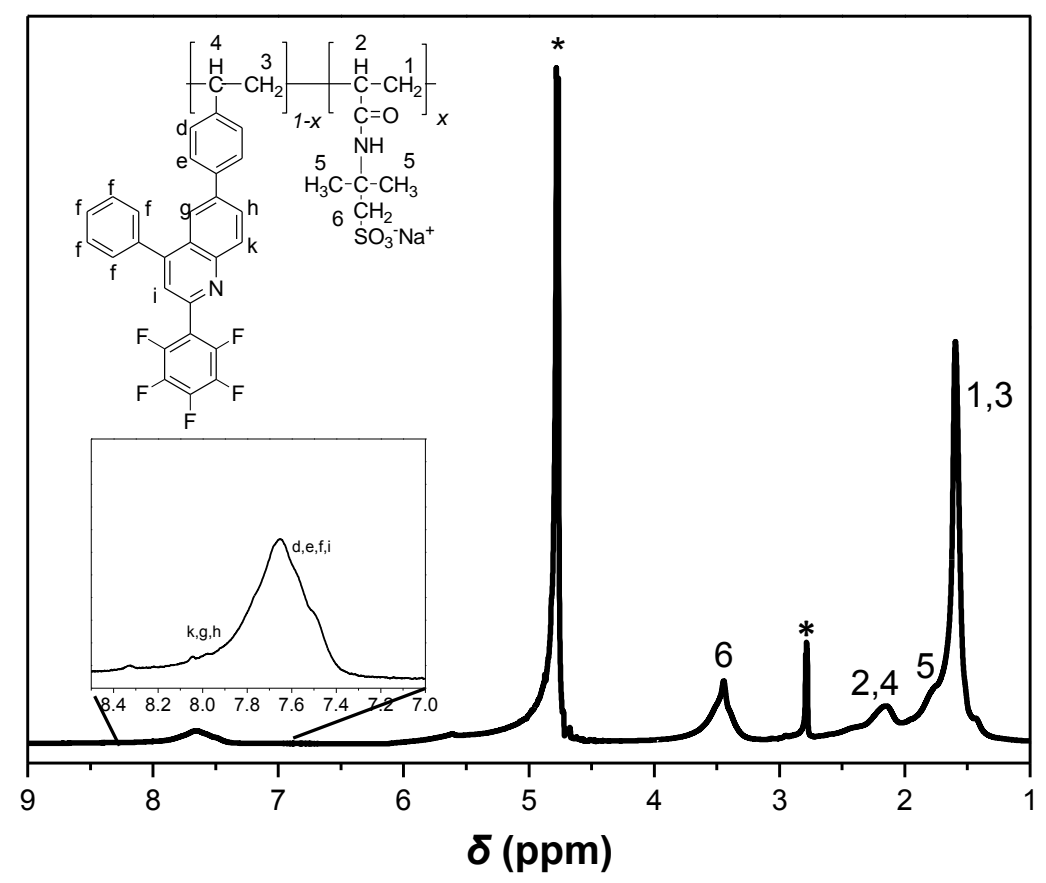

Figure S4. ${ }^{1} \mathrm{H}$ NMR spectrum of the copolymer $\mathrm{P}(\mathrm{AMPSNa}-\mathrm{co}-5 \mathrm{FSPQ})$ in $\mathrm{D}_{2} \mathrm{O}$. 


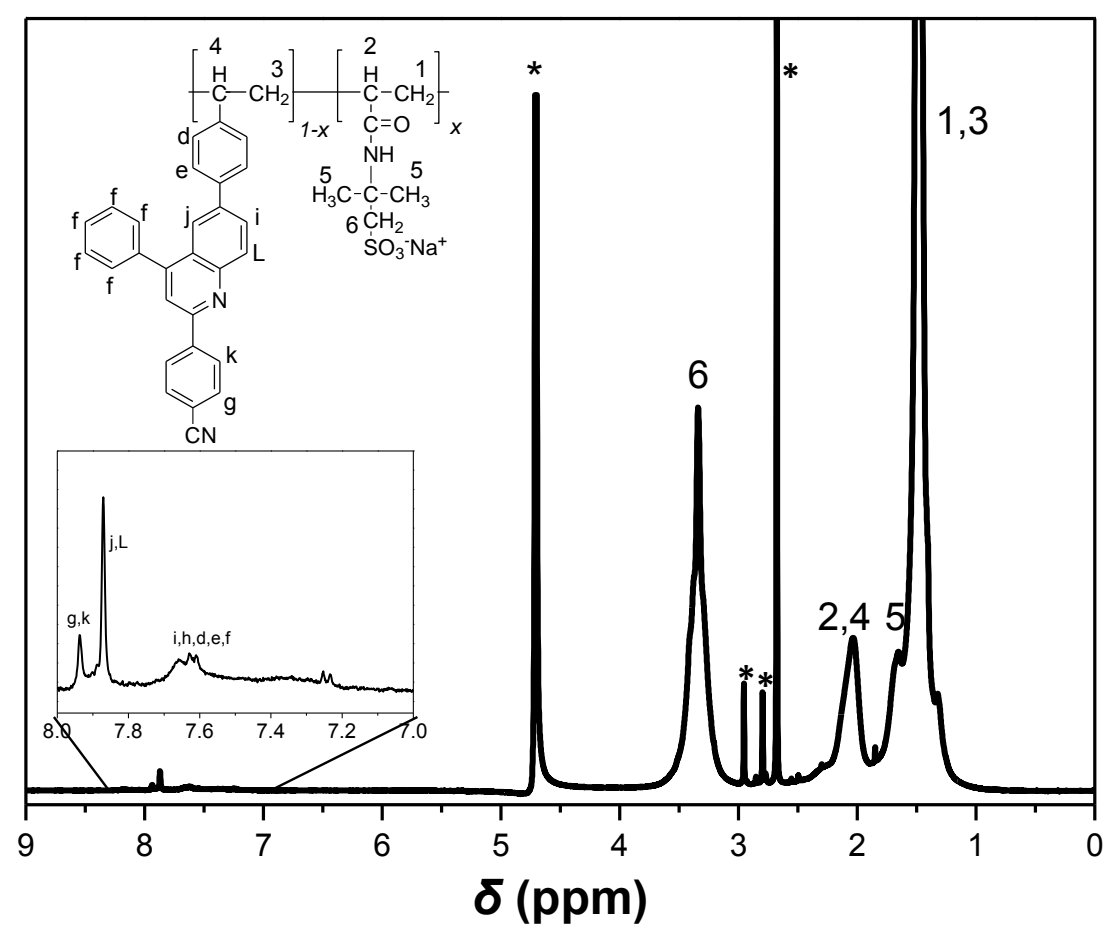

Figure S5. ${ }^{1} \mathrm{H}$ NMR spectrum of the copolymer $\mathrm{P}\left(\mathrm{AMPSNa}\right.$-co-SDPQCN) in $\mathrm{D}_{2} \mathrm{O}$.

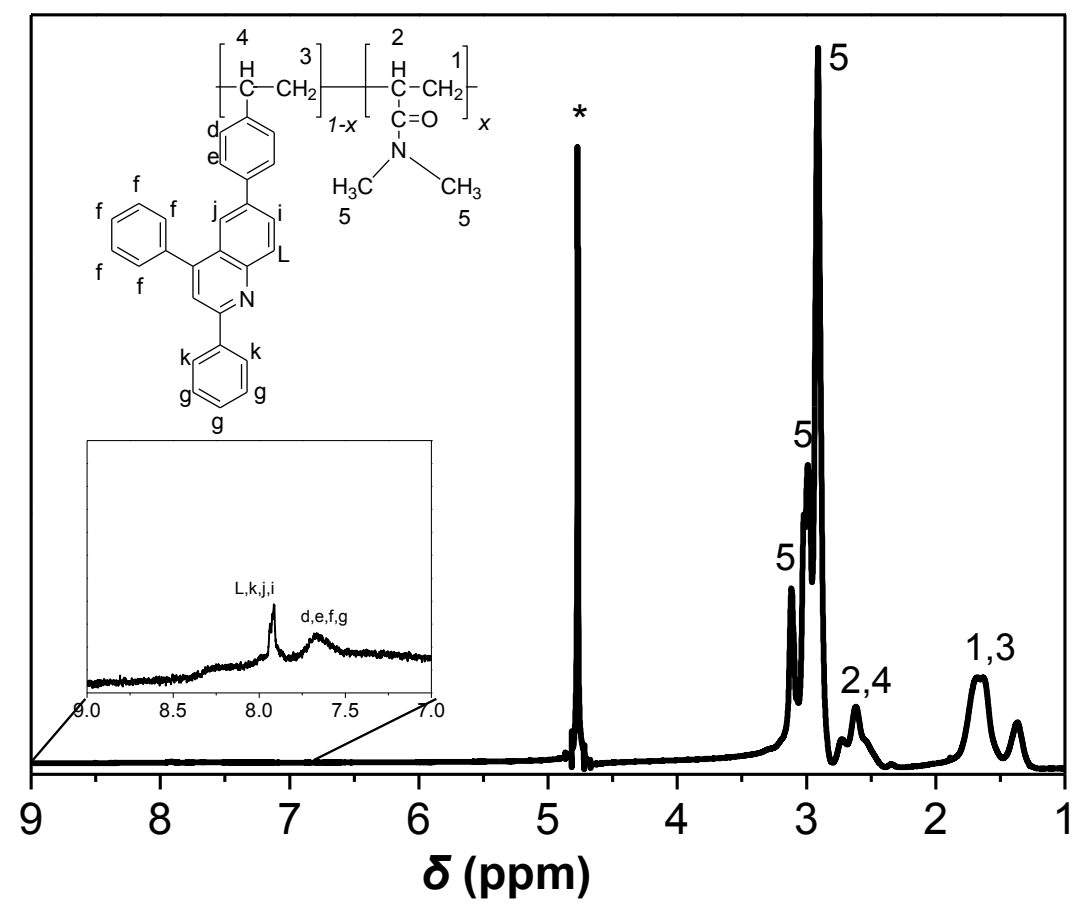

Figure S6. ${ }^{1} \mathrm{H}$ NMR spectrum of the copolymer $\mathrm{P}(\mathrm{DMAM}-\mathrm{co}-\mathrm{SDPQ})$ in $\mathrm{D}_{2} \mathrm{O}$. 


\section{Determination of the quinoline content of the copolymers through UV-Vis spectroscopy}

In order to determine the SDPQ content of the final products, the UV spectroscopy technique was enabled. Specifically, the SDPQ monomer was selected as reference molecule. Various solutions of known concentration were prepared, using THF as solvent and their absorption was monitored in order to build a calibration curve as it is presented in Figure S7. Then the copolymer P(DMAM-co-SDPQ) was dissolved in the same solvent and its SDPQ content was determined by the Beer-Lambert equation $(\mathrm{A}=\mathrm{ebC})$ of the calibration curve. The SDPQ content of the copolymer was found equal to $0.18 \mathrm{~mol} \%$.

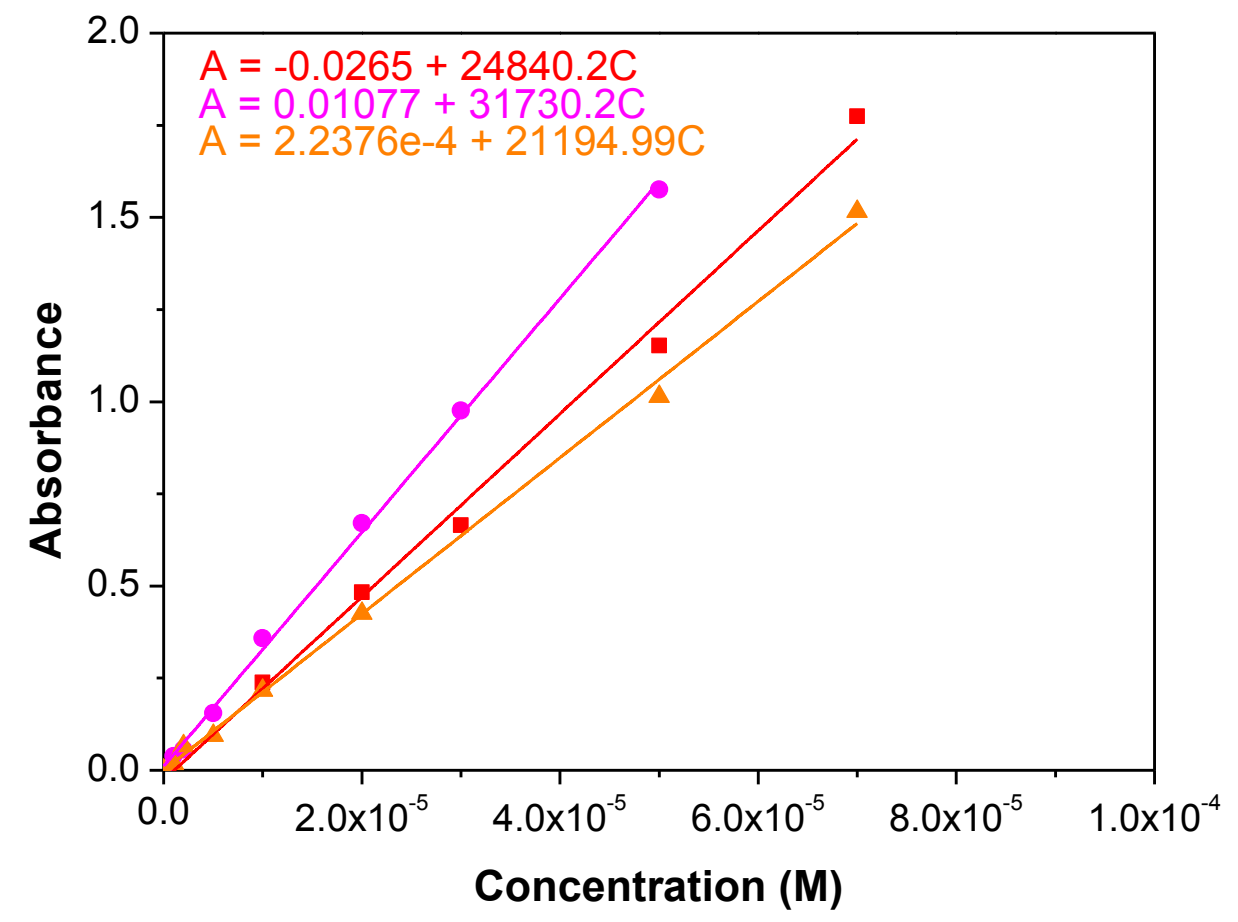

Figure S7. Calibration curves of the SDPQ (( $\bullet)$ red), SDPQCN ((•) magenta), 5FSPQ (( $\triangle)$ orange) used for the determination of the quinoline content of the P(DMAM-co-Quinoline) copolymers in THF. 
The quinoline content $\left(\lambda_{\mathrm{Q}}\right)$ in $\mathrm{g} / \mathrm{L}$ of the polymers can be obtained by taking the ratio of quinoline concentration in mol/L obtained by $\mathrm{UV}-\mathrm{V}$ is absorption measurements after applying Beer-Lambert law with $\varepsilon_{\mathrm{Q}}$ and the massic polymer concentration [Poly] in $\mathrm{g} / \mathrm{L}$.

$$
\lambda_{Q}=\frac{[Q]}{[\text { Poly }]}
$$

The molar fraction $(x)$ of quinoline-labeled monomers can be determined from equation:

$$
x=\frac{M_{o}}{1 / \lambda_{Q}+M_{o}-M_{Q}}
$$

where $\mathrm{Mo}$ and $\mathrm{M}_{\mathrm{Q}}$ are the molar masses of monomers used to prepare a given polymer and the quinoline-labeled monomer, respectively

Knowing the exact SDPQ content of this copolymer, various solutions of known concentration were prepared, using $\mathrm{H}_{2} \mathrm{O}$ as solvent and their absorption was monitored in order to build a calibration curve as it is presented in Figure S8. This step was performed in order to avoid solubility issues of the charged copolymers in organic solvents like THF. 


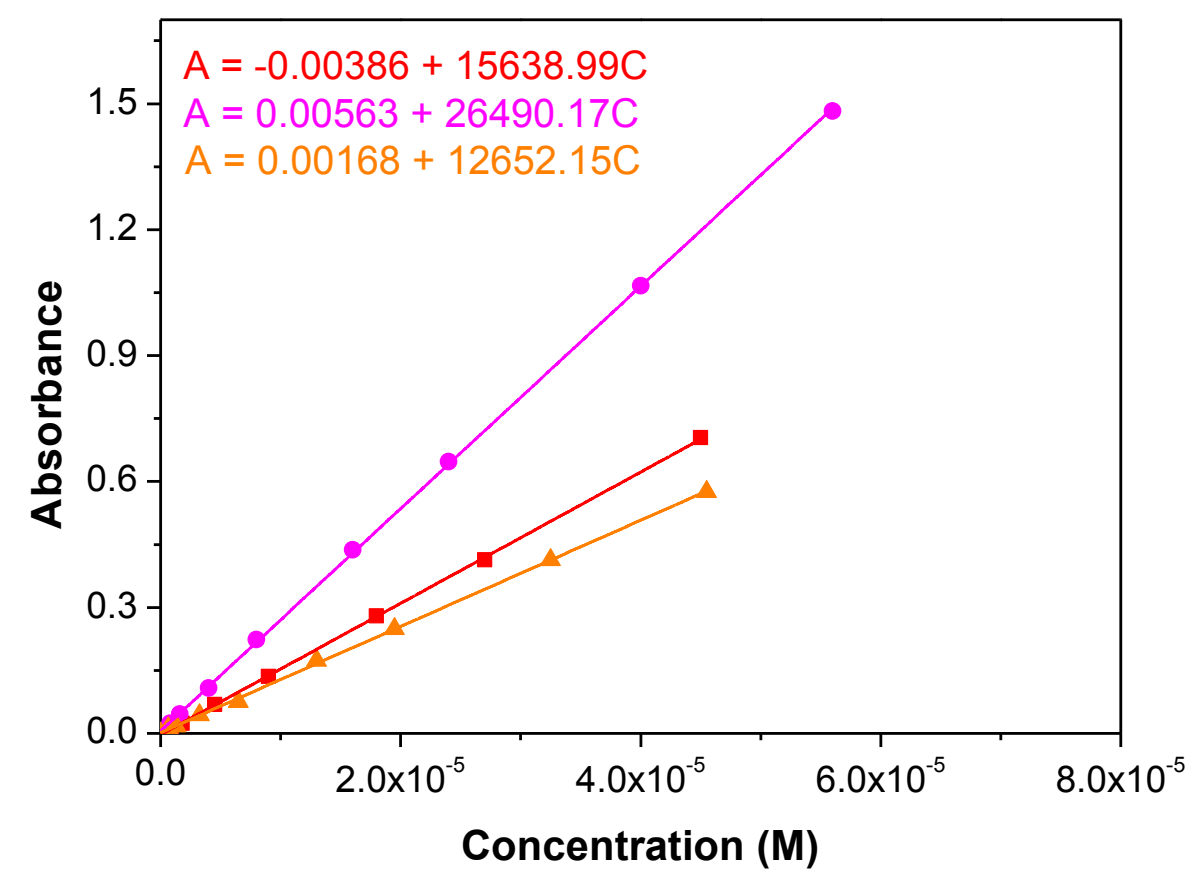

Figure S8. Calibration curves of the P(DMAM-co-SQPQ) (( $\square)$ red), P(DMAM-co-SDPQCN) $((\bullet)$ magenta), $\mathrm{P}(\mathrm{DMAM}-\mathrm{co}-5 \mathrm{FSPQ})\left((\triangle)\right.$ orange) copolymers in $\mathrm{H}_{2} \mathrm{O}$, used for the determination of the final quinoline content of the water-soluble quinoline- labeled polyelectrolytes.

The extinction coefficients as determined from Figures S7 and S8 are summarized in Table S1.

Table S1. Molar Extinction coefficients for the Quinoline monomers and the DMAM-based copolymers

\begin{tabular}{cc}
\hline Polymer & $\begin{array}{c}\text { Molar Extinction Coefficient } \\
\varepsilon\left(\mathrm{mol}^{-1} . \mathrm{L}_{\mathrm{cm}}{ }^{-1}\right)\end{array}$ \\
\hline SDPQ & 24800 \\
SDPQCN & 31700 \\
5FSPQ & 21200 \\
P(DMAM-co-SDPQ) & 15600 \\
P(DMAM-co-SDPQCN) & 26500 \\
P(DMAM-co-5FSPQ) & 12700 \\
\hline
\end{tabular}




\section{SEC characterization}

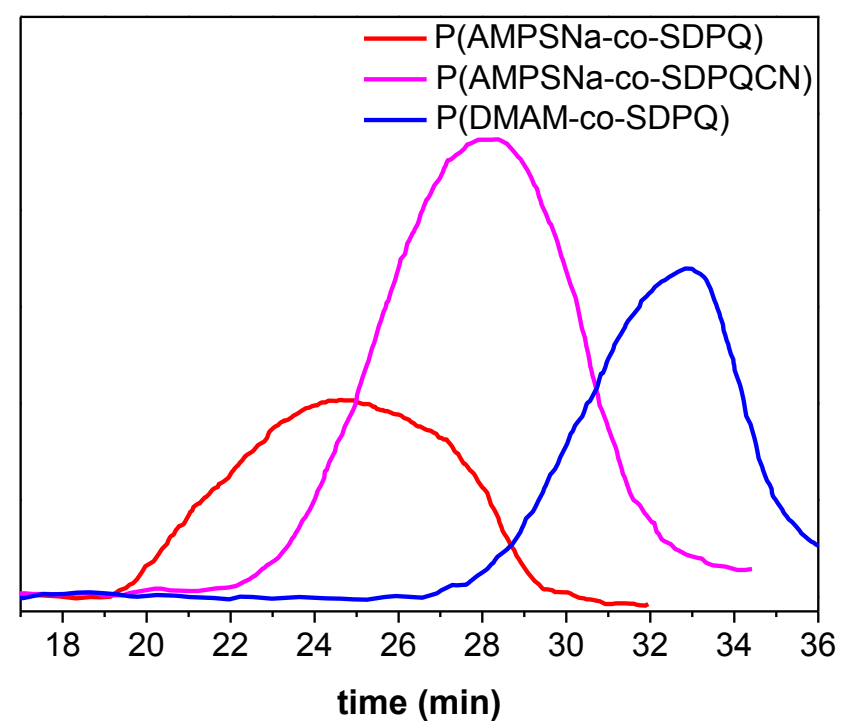

Figure S9: SEC curves of the P(AMPSNa-co-SDPC), P(AMPSNa-co-SDPQCN), P(DMAM-co-SDPQ) copolymers in $0.1 \mathrm{M} \mathrm{LiNO}_{3}$ as eluent. 


\section{UV-Vis absorption spectroscopy}

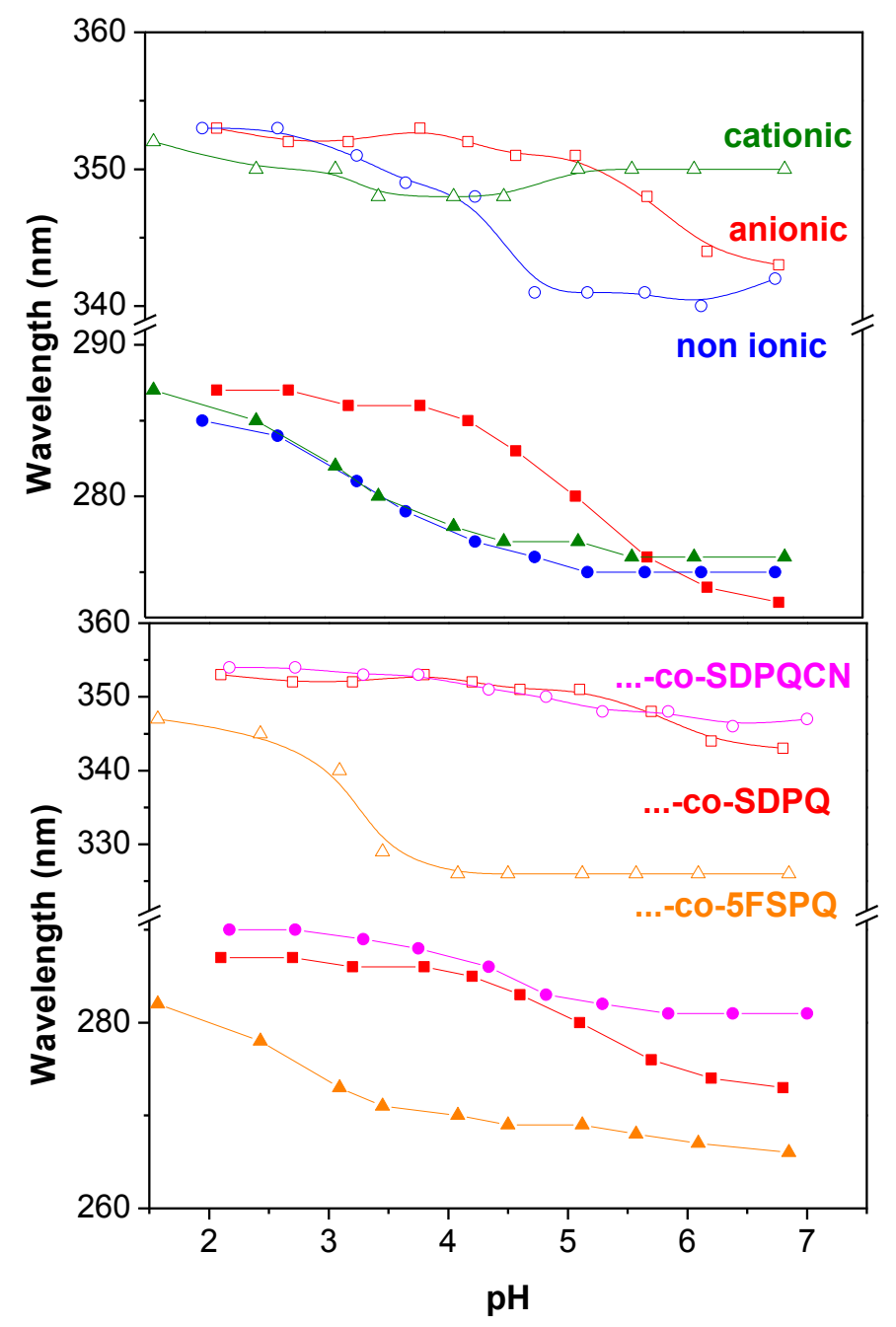

Figure S10. The position of the peaks for the three characteristic wavelengths $280 \mathrm{~nm}$ (solid), $350 \mathrm{~nm}$ (dashed) and 390nm (dotted), for the quinoline-labeled copolymers P(AMPSNa-co-SDPQ) ( $\square$ ), P(DMAM-co-SDPQ) (•), P(MAPTAC-co-SDPQ) ( $\Delta$ ), P(AMSPNa-co-SDPQCN) (•), P(AMPSNa-co$5 \operatorname{FSPQ})(\Delta) . \mathrm{C}_{\mathrm{Q}}=2 \times 10^{-5} \mathrm{M}$. 


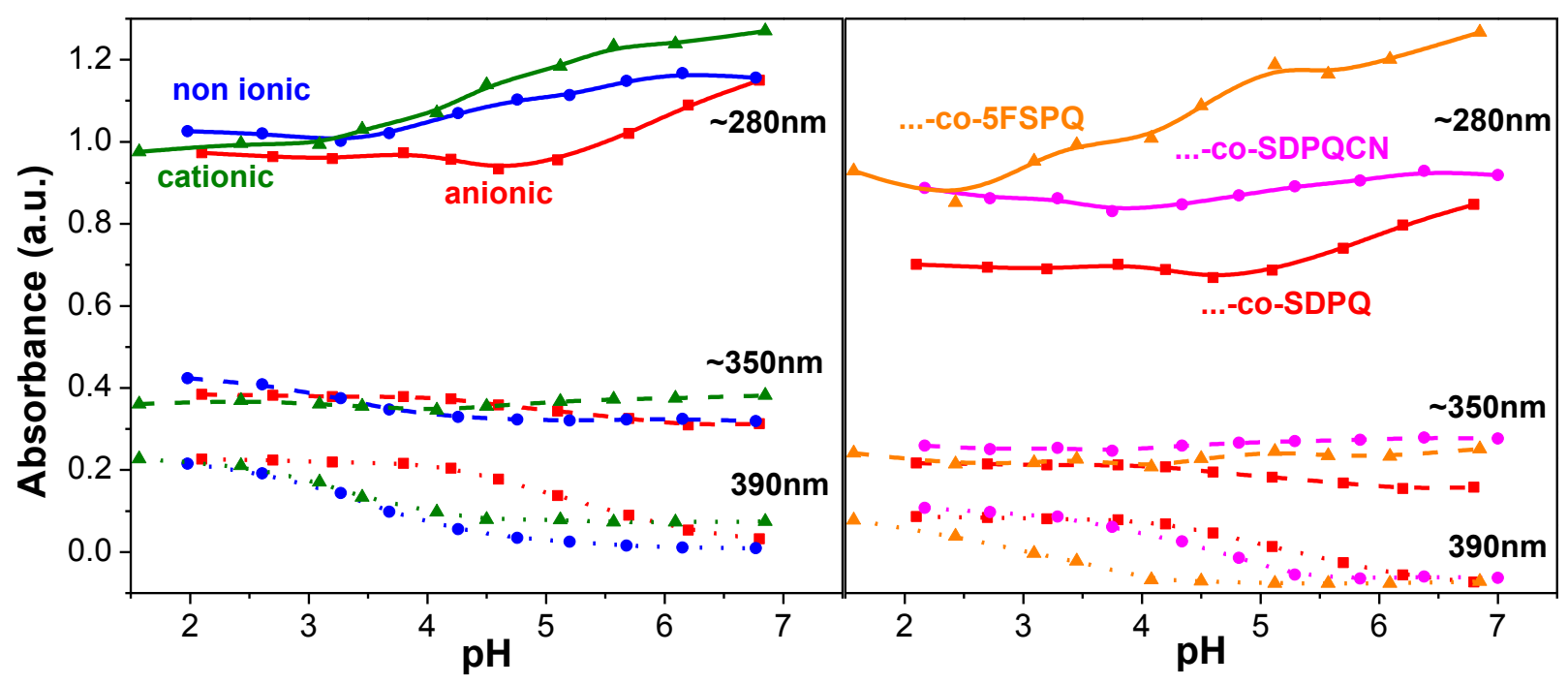

Figure S11. Maximum absorption for the three characteristic wavelengths $280 \mathrm{~nm}$ (solid), $350 \mathrm{~nm}$ (dashed) and 390nm (dotted), for the quinoline-labeled copolymers P(AMPSNa-co-SDPQ) ( $\square$ ),

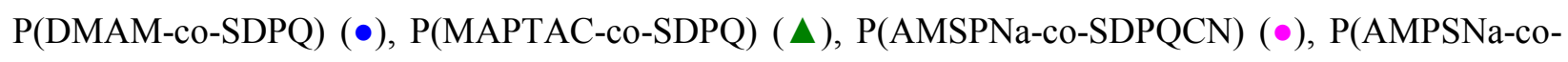
$5 \operatorname{FSPQ})(\Delta) . \mathrm{C}_{\mathrm{Q}}=2 \times 10^{-5} \mathrm{M}$. 


\section{Photoluminescence Spectroscopy}

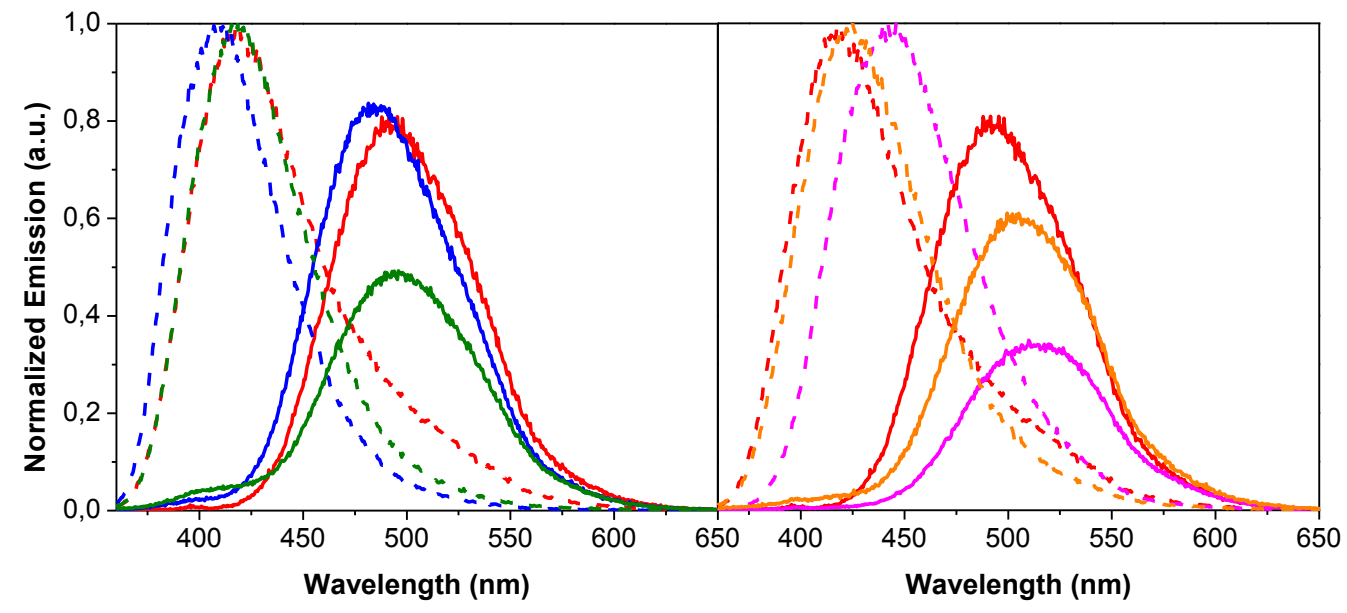

Figure S12. Emission spectra at $\mathrm{pH} \sim 2$ (solid lines) and at $\mathrm{pH} \sim 7$ (dashed lines) for the water-soluble copolymers P(AMPSNa-co-SDPQ) (red), P(DMAM-co-SDPQ) (blue), P(MAPTAC-co-SDPQ) (green), P(AMSPNa-co-SDPQCN) (magenta), P(AMPSNa-co-5FSPQ) (orange), upon excitation at 350nm. $\mathrm{C}_{\mathrm{Q}}=2 \times 10^{-6} \mathrm{M}$.

\section{Quantum Yields}

The quantum yield of the final water-soluble copolymers was determined using quinine as standard molecule. Quinine, when dissolved in $0.1 \mathrm{M} \mathrm{H}_{2} \mathrm{SO}_{4}$, has $\mathrm{Q}_{\mathrm{R}}=0.557$ when excited at $350 \mathrm{~nm}$, a wavelength very close to the second absorbance band of quinoline derivatives. The quantum yield of the copolymers was determined using the equation:

$$
Q=Q_{R} * I /_{I_{R}} * E_{R} /_{E} * n_{1} / n_{2}
$$


where $\mathrm{Q}=$ Quantum Yield, $\mathrm{I}=$ intergraded intensity, $\mathrm{E}=$ Absorbance and $\mathrm{n}=$ refraction index, while R stands for the standard (quinine).

The absorption and emission spectra, used for the calculations, are depicted at Figure S13 and Figure S14, respectively.

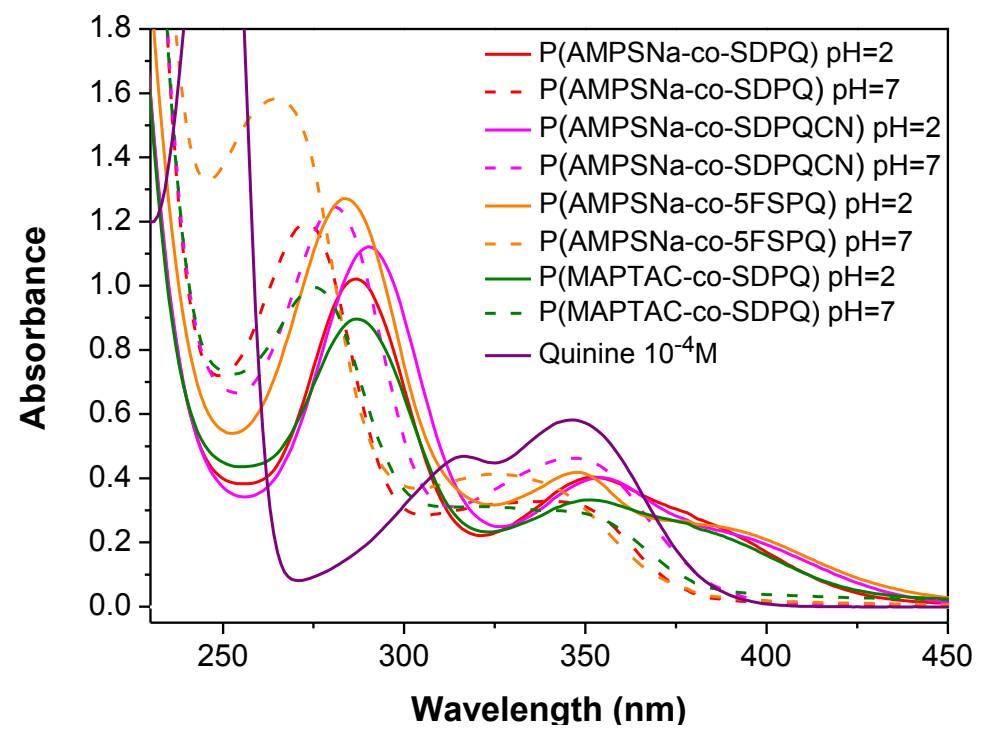

Figure S13. Absorption spectra of the water soluble copolymers at $\mathrm{pH} \sim 2$ and $\mathrm{pH} \sim 7$, as well as the absorption spectrum of quinine, used as standard in order to calculate the quantum yield of the quinoline unit. $\mathrm{C}_{\mathrm{Q}}=2 \times 10^{-5} \mathrm{M}$. 


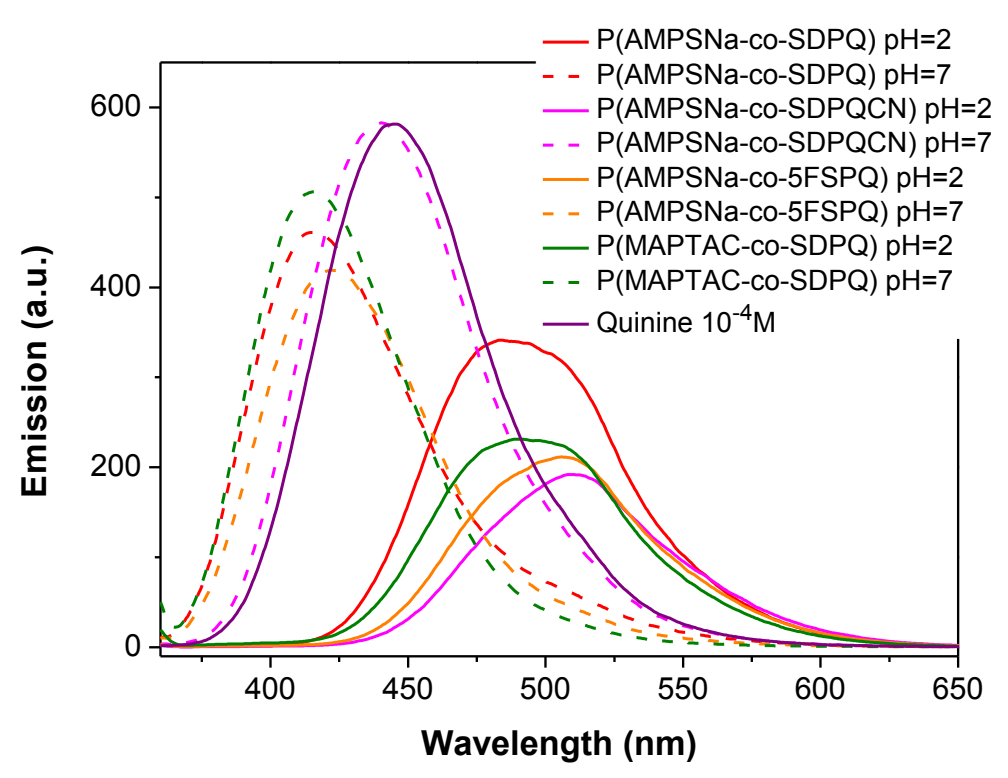

Figure S14. Emission spectra upon excitation at $350 \mathrm{~nm}$ of the water soluble copolymers at $\mathrm{pH} 2$ and $\mathrm{pH} \sim 7$ as well as the emission spectrum of quinine, used as standard in order to calculate the quantum yield of the quinoline unit. $\mathrm{C}_{\mathrm{Q}}=2 \times 10^{-6} \mathrm{M}$. 


\section{Nile Red probing}

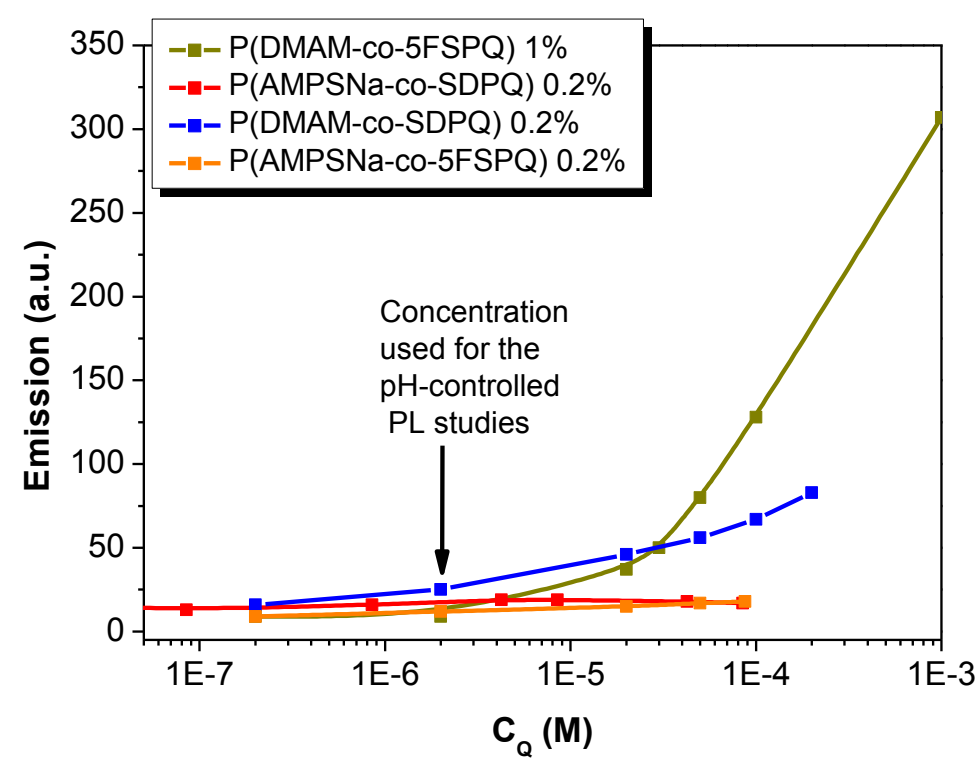

Figure S15: Nile Red probing results. The concentration of Nile Red is $1.6 \times 10^{-6} \mathrm{M}$ and the excitation wavelength is set at $550 \mathrm{~nm}$.

Table S2. Molar and mass concentrations of all quinoline- labeled copolymers synthesized in the present study.

\begin{tabular}{|c|c|c|c|}
\hline \multirow[b]{2}{*}{ Polymer } & \multirow{2}{*}{$\begin{array}{c}\text { Polymer } \\
\text { Concentration } \\
\text { for UV-Vis } \\
\text { Study }(\mathrm{g} / \mathrm{ml})\end{array}$} & \multicolumn{2}{|c|}{$\begin{array}{l}\text { Quinoline Concentration } \\
\text { for UV-Vis Study (M) }\end{array}$} \\
\hline & & $\begin{array}{c}\text { Based on Feed } \\
\text { composition } \\
(0.2 \mathrm{~mol} \% \\
\text { Quinoline })\end{array}$ & $\begin{array}{c}\text { Based on } \\
\text { UV-Vis } \\
\text { Calibration } \\
\text { curves }\end{array}$ \\
\hline P(AMPSNa-co-SDPQ) & 0.00229 & $210^{-5}$ & $2.2 \times 10^{-5}$ \\
\hline P(AMPSNa-co-SDPQCN) & 0.00229 & $210^{-5}$ & $2 \times 10^{-5}$ \\
\hline P(AMPSNa-co-5FSPQ) & 0.00229 & $210^{-5}$ & $3 \times 10^{-5}$ \\
\hline P(DMAM-co-SDPQ) & 0.000996 & $210^{-5}$ & $1.8 \times 10^{-5}$ \\
\hline P(MAPTAC-co-SDPQ) & 0.00221 & $210^{-5}$ & $1.8 \times 10^{-5}$ \\
\hline
\end{tabular}

